\title{
CrimRxiv
}

\section{Review 1 of "Victim refuses to cooperate: A mixed methods examination of complainant cooperation in sexual assault cases involving adolescent victims"}

Published on: Dec 22, 2020

DOI: $10.21428 / c b 6 a b 371 . a 00 f a 86 a$

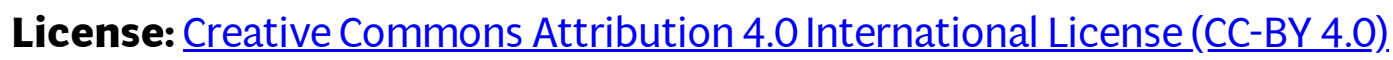


Vote: Reject

\section{[Please explain your vote. If you voted to publish pending minor changes, specify each change, why it is needed, and, possibly, how it should/could be done.]}

Thank you for allowing me to review the manuscript, "Victim refuses to cooperate: A mixed methods examination of complainant cooperation in sexual assault cases involving adolescent victims". While this paper is well-written, uses appropriate data, and provides a strong overview of the prior research, the authors build their study on an undemonstrated contention: adolescent victims of sexual assault decline to cooperate for reasons that are different than adult victims (and thus require special consideration). While this argument is valid on its face, given that the authors drew their subsample from a dataset including both adult and adolescent victims of sexual assault, the study should first establish these differences (and then explore any differences in the qualitative data). Indeed, the authors note in the discussion that: "our results support arguments that future case processing research should...work toward identifying whether cooperation decisions and reasons for withdrawal vary by complainant age".

As it stands, the discussion could better tie the findings to extant literature. The 3 strongest predictors were (1) perceived character issues, (2) threats/use of weapons, and (3) minority suspects. All of these have been previously tied to victim cooperation decision-making, but where do the direction of these relationships align and diverge from prior research adult sexual assault (and on adolescents)? For example, based on the prior literature we would expect character issues to decrease and seriousness of the crime to increase victim cooperation (as seen in these results), but no prior studies are linked and discussed. Further, these findings seem to show similarities between adult and adolescent cases.

Regarding the two significant age-related factors: suspect age and parental witnesses, I would encourage the authors to further examine these analyses and findings. First, the authors present a positive relationship between suspect older age and cooperation in the results/table, but then indicate the opposite relationship in the discussion. Either way, suspects in these adolescent cases were 27 years old on average, while victims were 15 years old. Thus, many of these cases are statutory rape, which might be treated differently by police than cases involving two adolescents. The phenomenon of 
statutory rape is unique to adolescents and it not discussed in the current paper. Did the authors examine this? If the discussion is correct, and suspect older age was associated with a decrease in victim cooperation, I would encourage the authors to more closely assess who these perpetrators are to the victims and how these relationships might impact cooperation. In other words, given that most cases were non-strangers might the relationships between these adults and these adolescents' families impact cooperation? Might these victims be fearful about how prosecution of these suspects might impact their families above and beyond any power differentials suggested in the discussion (in the same and/or different ways we see in adult victims)? Suspect age might be serving as a proxy variable for something unique to adolescent cases that requires further investigation. Further, might parents/relatives simply be seen as credible witnesses with no greater (or lesser) impact on victim cooperation than cases involving other credible witnesses? Finally, parent/relative reported assault to the police was not significant as predicted, but this was not addressed in the discussion.

Research inclusive of adolescent victims of sexual assault is important; however, providing baseline analyses across adolescent and adult victims first, and then exploring any true "differences" would provide for a stronger analysis of whether and/or how adolescent victims are different than adult victims in regard to their cooperation decisions. 